\title{
Coordinated Path Planning for Surface Acoustic Beacons for Supporting Underwater Localization
}

\author{
Anwar Quraishi, Alcherio Martinoli
}

\begin{abstract}
Accurate localization is one of the biggest challenges in underwater robotics. The primary reasons behind that are unavailability of satellite-based positioning below the surface, and lack of clear features in natural water bodies for visually aided localization. As such, the common method of choice for external position referencing in underwater robots is the use of acoustic signals for computing range or direction of arrival. To that end, we have developed an acoustic range based navigation system with floating, movable beacons. In this paper, we present an approach for planning the trajectory of acoustic beacons in a way that they provide the best possible navigation support for a group of underwater vehicles. We use an information theoretic approach to beacon path planning that minimizes the group's position uncertainty. We evaluate our approach with realistic simulations calibrated using real-world data, and present results.
\end{abstract}

\section{INTRODUCTION}

The physical properties of water limit application of common outdoor robot localization methods to underwater robots. The opacity of water to electromagnetic waves renders satellite-based positioning as well as radio communication unusable. Further, vision-based methods are not reliable due to turbidity or lack of proximity to physical features in natural water bodies. Highly accurate inertial sensors may offer acceptable positioning accuracy, but are expensive and often not compatible with small-sized underwater robots. A common approach to underwater localization and communication, therefore, is to employ acoustic signals [1]. The time of arrival of an acoustic signal is then used to either compute range (based on time-of-flight) or direction of arrival (with a multi-receiver array) to a transmitting beacon, whose position must be known. A series of such geometric measurements are used to deduce underwater position. Accurate on-board positioning is necessary for underwater robots to follow a pre-planned trajectory, and to provide an accurate georeference for any spatial data collected.

We are developing Autonomous Underwater Vehicles (AUVs) for environmental sensing in lakes and coastal areas [2]. We have previously presented a navigation system using surface beacons at known positions that provide a series of range measurements to aid underwater localization [3]. The beacons could transmit their own positions using acoustic communication, and therefore did not need to be stationary.

Distributed Intelligent Systems and Algorithms Laboratory (DISAL), School of Architecture, Civil and Environmental Engineering, École Polytechnique Fédérale de Lausanne (EPFL), 1015 Lausanne, Switzerland. This work was partially funded by the Swiss National Science Foundation under grant CRSII2_160726/1. https://disal.epfl.ch/research/AUVDistributedSensing.

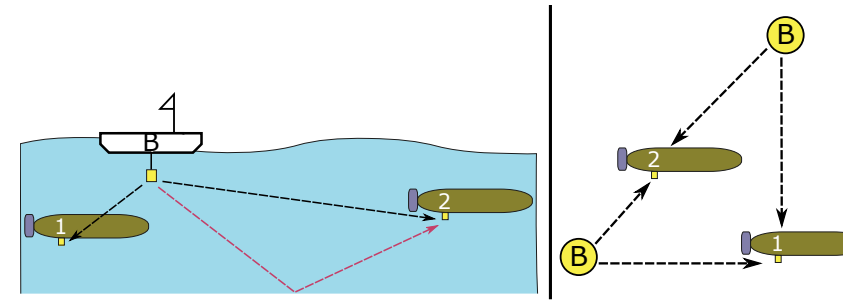

Fig. 1: Schematic showing effect of beacon placement, and reflections with long distances.

However, the motion of the robots and beacons was uncoordinated, which poses a number of drawbacks. First, the accuracy of range measurements decreases with increasing distance, as we will show later. This is because the acoustic signal strength drops as a square of distance, making the received signal susceptible to noise from the environment and the motors. The occurrence of outliers due to echoes and reflections also increases with increasing distance. Second, certain relative position configurations (such as collinearity between two beacons and a robot) provide range measurements resulting in position updates with high error. This is illustrated in Fig. 1.

In this paper, we present an actively controlled beacon system for providing localization support. Each beacon is mounted on an Autonomous Surface Vehicle (ASV), whose trajectory is controlled in a way that the aforementioned problems are avoided. Further, they are commanded to move to a position that provides the underwater robots with the highest possible gain in position information.

Much of the existing work in underwater acoustic localization utilizes beacons deployed at a known location, usually on the surface [1], [4]. Becker et. al. in [5] presented an approach inspired by Simultaneous Localization and Mapping (SLAM) to localize both the robot and a single fixed beacon with unknown position but known depth. By using synchronized clocks, range measurements can be obtained from a single acoustic transmission with known transmission time, as opposed to a round trip exchange. In [6], a multi-channel receiver array was installed on the robots and synchronized clocks were used to perform range and bearing measurements with one-way signal transmission from a single beacon. The beacon was fixed. Guo et. al. in [7] perform localization considering that an anchored beacon can sway. However, beacon mobility is not actively controlled. In [8], the authors implement an acoustic network that also provides timing information to enable range measurements from one-way transmissions. They also recognize that fixed beacons limit 
the operational area of the robots. In our previous work [3], the beacons were mounted on surface robots, making them mobile. However, mobility was only used for easy deployment and not actively controlled to extend the area of operation. All the aforementioned approaches suffer from the drawbacks outlined earlier.

In [9], AUVs equipped with a multi-receiver array measure range and bearing to a beacon. They use this to localize themselves and also maintain a fixed relative position with respect to the beacon. Then, the AUVs move in response to motion of the beacon, effectively sidestepping the problem of limitation in operational area. However, it is the beacon that influences the AUV motion in a centralized fashion, not the other way around.

In contrast, we seek to develop a method for surface acoustic beacons to move automatically in response to the localization needs of AUVs, in a way that the drawbacks outlined earlier are avoided. In a similar vein, several approaches have previously been developed for localizing fixed sensor nodes at unknown locations in a Wireless Sensor Network (WSN). Erdemir et.al. [10] proposed a method where a mobile beacon traverses an optimal path that provides sufficient range measurements to the fixed nodes for localization by trilateration. Note that here the beacon is mobile, while the localization targets - the sensing nodes are static at unknown positions. Other approaches addressing the same problem have been presented in [11], [12], [13]. The distinction in the robotics problem is that both the beacon and the localization targets - the AUVs - are mobile, and the positions of the AUVs are not completely unknown.

We consider AUVs and beacons equipped with acoustic transceivers that can also calculate range based on time-offlight, and broadcast their positions. The beacons, being on the surface, obtain their position from a Global Navigation Satellite System (GNSS), while the AUVs estimate their position by fusing inertial measurements with range measurements. The AUVs perform environmental sampling, or any other activity autonomously. The AUVs also periodically broadcast their estimated position. Then, at each time instant, we calculate the future desired position of the beacons such that the next cycle of updates results in the maximum reduction in collective uncertainty in AUVs' positions, and the beacons remain sufficiently close the the group of AUVs.

We tested our approach with realistically simulated experiments. The simulations were calibrated to the real-world performance of acoustic transceivers. We show that our approach for path planning of mobile beacons improves the localization accuracy in comparison to static beacons as well as mobile beacons moving in a fixed formation.

\section{Preliminaries and System Components}

We consider deployment of a team of AUVs for any cooperative activity, for example, environmental sampling. The AUVs plan their own path, possibly adapting it during operation. The only assumption we make about AUV trajectories is that they are always within a distance of $d_{\max }$ from each other such that $d_{\max }<r_{\max }$, where $r_{\max }$ is the maximum

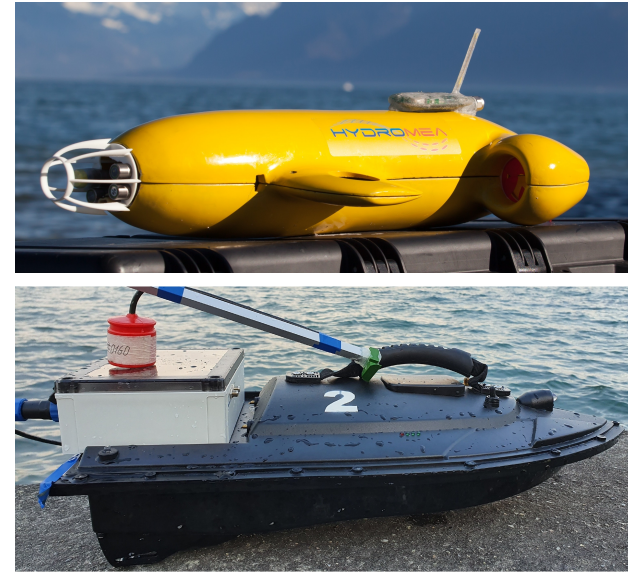

Fig. 2: Above: The Vertex AUV with its sensor suite in the front. The AUV is about $70 \mathrm{~cm}$ long, weighs $7 \mathrm{~kg}$. Below: The ASV with its acoustic transceiver. The ASV is about $50 \mathrm{~cm}$ long and weighs around $2.4 \mathrm{~kg}$.

range of the acoustic transceivers. We also deploy mobile acoustic beacons mounted on ASVs. The beacons have no prior knowledge of the motion of the AUVs. Our goal is to estimate optimal positions for the beacons in real-time. This section introduces the robotic platforms considered in this work and relevant subsystems.

\section{A. Robots}

We consider two different kinds of robots in this work - ASVs and Vertex AUVs [2]. The AUV is a small, light weight $(70 \mathrm{~cm}, 7 \mathrm{~kg})$ platform equipped with a suite of environmental measurement probes. The ASV is $80 \mathrm{~cm}$ in length and weighs $2.4 \mathrm{~kg}$. The robots are shown in Fig. 2. The AUV and ASVs have the same sensing and computation hardware, consisting of an Inertial Measurement Unit (IMU), an acoustic transceiver and a GNSS receiver. On the AUV, the GNSS receiver works only when it is not submerged, while ASVs benefit from continuous GNSS and radio communication.

In this paper, the ASVs function as mobile acoustic beacons and provide localization support to the AUVs. By communication their own position obtained from GNSS using the acoustic transceivers, they provide range measurements from a known reference point.

\section{B. Position estimation}

An Extended Kalman Filter (EKF) is used for state estimation, including position. The state comprises of position, velocity and acceleration:

$$
\mathbf{X} \triangleq\left[\vec{x}^{\mathrm{T}}, \vec{v}^{\mathrm{T}}, \vec{a}^{\mathrm{T}}\right]^{\mathrm{T}}
$$

The process model takes into account propeller thrust as well as hydrodynamic drag forces for inertial positioning.

$$
\begin{aligned}
\hat{\mathbf{X}}^{t+\delta t} & =f\left(\mathbf{X}^{t}, \delta t, U^{t}\right), \\
\hat{\Sigma}^{t+\delta t} & =F \Sigma^{t} F^{\mathrm{T}}+R,
\end{aligned}
$$

where $f$ is the process model and $F$ is the corresponding Jacobian matrix, $\mathbf{X}^{t}$ is the state (including position $\vec{x}^{t}$, velocity and acceleration), $\Sigma^{t}$ is the state covariance and $U^{t}$ is the 


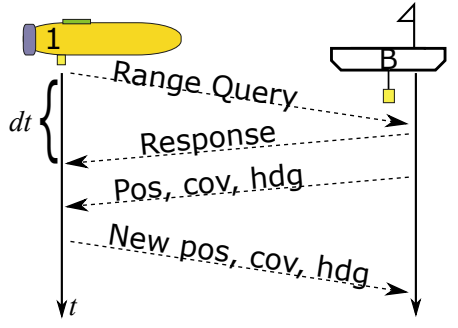

Fig. 3: Exchange of acoustic signals and information during a range measurement. The robots transmit their 2D position, covariance (3 covariance matrix elements corresponding to $2 \mathrm{D}$ position) and heading.

control input, all at time $t . R$ is the (constant) covariance matrix describing the motion noise, which is determined empirically. IMU, GNSS, acoustic or depth measurements are fused when they become available using standard EKF equations. We will see later that the process model is also used by the beacons to predict the AUVs' future positions.

\section{Acoustic ranging and communication}

All the robots are equipped with DiveNET Microlink acoustic transceivers that are capable of exchanging data as well as measuring range. The data throughput is $80 \mathrm{bits} / \mathrm{s}$ and the communication is broadcast. All robots can listen to any transmitted signal. Range measurements are performed using a query-response cycle and measuring the two-way time-offlight. Accuracy characteristics are shown later in the paper.

Along with the range query, the AUVs and the beacons exchange their position, 2D position covariance and heading as shown in Fig. 3. To avoid overlapping range queries and signal collisions, AUVs are assigned fixed time-slots of $4 \mathrm{~s}$ duration during which they can initiate a range query, a scheme known as Time Division Multiple Access (TDMA). We have implemented such a scheme in a real system in [3].

\section{Range updates}

Range measurements are fused into the position estimates using the EKF framework. Given the estimated state of the AUV $\hat{\mathbf{X}}$, and covariance $\hat{\Sigma}$, as well as the position of the beacon $\vec{x}_{\mathrm{b}}$, the measurement function is

$$
g(\hat{\mathbf{X}})=\left\|\hat{x}-\vec{x}_{\mathrm{b}}\right\| .
$$

The Jacobian of the measurement model can be obtained as

$$
G=\frac{\partial g(\hat{\mathbf{X}})}{\partial \hat{\vec{x}}}=\left[\frac{1}{g(\hat{\mathbf{X}})} \cdot\left(\hat{x}-\vec{x}_{\mathrm{b}}\right)^{\mathrm{T}}, 0,0, \ldots\right] .
$$

Using the standard EKF equations, the updated position and covariance of each AUV is calculated as follows (note that we dropped the time superscript).

$$
\begin{aligned}
K & =\hat{\Sigma} G^{\mathrm{T}}\left[G \hat{\Sigma} G^{\mathrm{T}}+\sigma_{r}\right]^{-1}, \\
\mathbf{X} & =\hat{\mathbf{X}}+K(z-g(\hat{\mathbf{X}})), \\
\Sigma & =(\mathrm{I}-K G) \hat{\Sigma},
\end{aligned}
$$

where $K$ is the Kalman gain, $z$ is the range measurement, $\sigma_{r}$ represents the range measurement noise, I is an identity matrix of appropriate dimension, and the bracketed inverse expression in Eq. (6) evaluates to a scalar.

While errors in range measurements cannot be accurately modeled with a Gaussian distribution, a Gaussian assumption works well in practice. It also offers a number of advantages such as low computational cost and simple implementation, which make it suitable for a real-time system.

\section{E. Problem statement}

Given a set of beacons and a set of AUVs, as the AUVs travel while carrying out their activity, we require that

1) the beacons move so that they are within a specified distance of $r_{\max }$ from all AUVs,

2) no AUV is collinear with the beacons, and

3) the beacon placement minimizes the posterior uncertainty in AUVs' positions.

As stated earlier, we assume that the AUVs are within a distance of $d_{\max }$ from each other.

\section{Methodology}

The beacons, mounted on ASVs, predict the future position and position covariance of the AUVs using the most recent position information broadcast by them. This is then used to compute an optimal placement for the beacons in the next few seconds.

\section{A. Position prediction}

The beacons employ the process model of the AUVs in Eqs. $(2,3)$ to predict the AUVs' position, $\hat{x}$ and uncertainty in position, $\hat{\Sigma}_{x}$ at a future point in time. They use the information broadcast by the AUVs and assume a constant speed of $1 \mathrm{~m} / \mathrm{s}$ (the AUV speed is set to this value). The control input is assumed such that the drag forces are canceled out. Note that since the AUVs can change their direction, this prediction only serves as a heuristic.

\section{B. Optimal beacon placement}

We aim to find the beacon location that would provide the best reduction in uncertainty in AUVs' positions after the range update. We do so by minimizing the collective posterior (differential) entropy. Let $\vec{x}_{\mathrm{b}}$ be the future position of the beacon, and let $X_{i}$ be the random variable describing the posterior position of AUV $i$ after the future range update. We seek to solve the following minimization problem:

$$
\vec{x}_{\mathrm{b}}^{*}=\underset{\vec{x}_{\mathrm{b}}}{\arg \min } h\left(X_{1}, X_{2}, \ldots\right),
$$

where $h($.$) is the differential entropy of a continuous distribu-$ tion. Here, we minimize the entropy of the joint distribution of posterior AUV positions. It is reasonable to assume that the AUV positions are independent, since they do not use each others' position estimates. Therefore, the joint entropy reduces to a sum of individual entropies.

$$
h\left(X_{1}, X_{2}, \ldots\right)=h\left(X_{1}\right)+h\left(X_{2}\right)+\ldots
$$

Further, given the Gaussian assumption, we have

$$
X_{i} \sim \mathcal{N}\left(\vec{x}_{i}, \Sigma_{x_{i}}\right)
$$




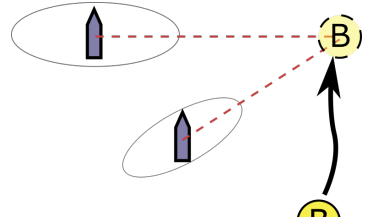

(B)

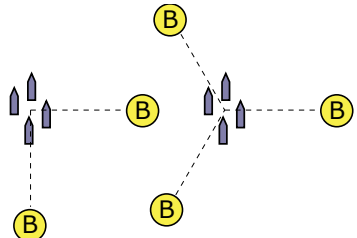

(b) Desired beacon distribution (a) Optimal placemen
Fig. 4: (a) Illustration of the optimal placement of the beacon given two AUVs and their position uncertainties. (b) Desired placement of several beacons around a group of AUVs. The ideal placement is a uniform distribution of beacons around the centroid of the group.

where $\vec{x}_{i}$ and $\Sigma_{x_{i}}$ are posterior position and positioncovariance of AUV $i$. Therefore, for $X_{i}$ of dimension $n$, the differential entropy $h\left(X_{i}\right)$ can be evaluated as [14]

$$
h\left(X_{i}\right)=\frac{1}{2} \log \left((2 \pi e)^{n}\left|\Sigma_{x_{i}}\right|\right) \text { bits, }
$$

The posterior covariance $\Sigma_{x_{i}}$ can be obtained by predicting the prior position and covariance at a future time, $\hat{x}_{i}, \hat{\Sigma}_{x_{i}}$ (see Section III-A), and then applying the Kalman update equation in Eq. (8) using the future potential range measurement. Following from Eqs. (4-6) and Eq. (8), we obtain

$\Sigma_{x_{i}}=\left(\mathrm{I}_{2 \times 2}-\frac{\hat{\Sigma}_{x_{i}}\left(\hat{x}-\vec{x}_{\mathrm{b}}\right)\left(\hat{x}-\vec{x}_{\mathrm{b}}\right)^{\mathrm{T}} / g(\hat{x})^{2}}{\left(\left(\hat{x}-\vec{x}_{\mathrm{b}}\right)^{\mathrm{T}} \hat{\Sigma}_{x_{i}}\left(\hat{x}-\vec{x}_{\mathrm{b}}\right) / g(\hat{x})^{2}\right)+\sigma_{r}}\right) \hat{\Sigma}_{x}$

The expression in the denominator here evaluates to a scalar.

Following from Eqs. (10), (12), the joint entropy of AUV positions is calculated as

$$
\begin{aligned}
h\left(X_{1}, X_{2}, \ldots\right) & =\sum_{i} \frac{1}{2} \log \left((2 \pi e)^{n}\left|\Sigma_{x_{i}}\right|\right) \\
& =\frac{1}{2} \log \prod_{i}\left((2 \pi e)^{n}\left|\Sigma_{x_{i}}\right|\right) .
\end{aligned}
$$

Using this, we can reformulate the optimization problem in Eq. (9) as

$$
\vec{x}_{\mathrm{b}}^{*}=\underset{\vec{x}_{\mathrm{b}}}{\arg \min } \prod_{i}\left|\Sigma_{x_{i}}\right| .
$$

In Eq. (13), note the dependence of $\Sigma_{x_{i}}$ on position of the beacon, $\vec{x}_{\mathrm{b}}$. This minimization is performed by doing a search on a uniform grid of granularity $1 \mathrm{~m}$ around the centroid of the predicted AUV positions.

For a single AUV, $\vec{x}_{\mathrm{b}}^{*}$ corresponds to any position along the direction of maximum prior uncertainty in the AUV's position estimate. With a Gaussian distribution, this is the line along the first eigenvector of the prior covariance matrix $\hat{\Sigma}_{x}$. For two AUVs, $\vec{x}_{\mathrm{b}}^{*}$ lies on the intersection of two such lines, one for each AUV. This is illustrated in Fig. 4.

\section{Placement of subsequent beacons}

After range updates to all AUVs from an optimally placed beacon (see Fig. 4a), the uncertainties in their positions change, resulting in a new optimal beacon location. Theoretically, one beacon can provide sufficient localization support by repeatedly moving to new optimal locations. However, in practice, each cycle of range updates may result

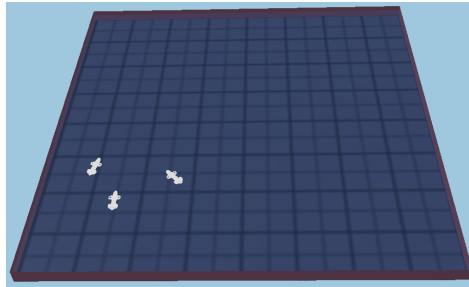

Fig. 5: A screenshot of the Webots simulation environment with three robots. Experiments are performed in a $140 \times 140 \mathrm{~m}$ arena

in large changes in the optimal beacon location, and a single beacon may not be able to carry out the amount of motion required. Further, additional beacons will improve localization by immediately providing range information along a complementary direction.

Given that range updates provide position information along only one direction, we ideally require additional range measurements from a diversity of directions. Therefore, the desired placement of subsequent beacons is such that they have a uniform circular distribution around the AUVs, as shown in Fig. 4b. In this paper, we considered the case of two beacons. We place the second beacon such that the two beacons are in perpendicular directions with respect to the centroid of the group of AUVs.

\section{Master and slave beacons}

We designate one of the beacons as the master, which computes and assigns future placement to itself and the slave beacon. Note that decentralized approaches are also possible, but we exploited the fact that the beacons, being on the surface, have access to radio communication.

The master beacon obtains the first desired position using Eq. (16) in Section III-B, and computes the second such that

- it is in a perpendicular direction with respect to the centroid of the AUV group (see Fig. 4),

- it does not cause collinearity with the other beacon and any of the AUVs to the extent possible, and

- it is within a distance of $r_{\max }$ from each AUV.

Then, the two positions are assigned to the closest ASVs in a way that the total travel distance is minimized.

\section{EXPERIMENTS}

The proposed method was implemented and tested with a group of one and three AUVs and one and two beacons. Experiments were performed in simulation, and the acoustic range measurements were calibrated to outdoor, real-world performance, both in terms of accuracy and measurement intervals. We evaluated the algorithm in terms of localization accuracy. We compared the proposed optimal placement approach with a variety of static and moving beacon configurations.

\section{A. Simulation}

We used Webots [15], a high-fidelity robotics simulation software. A picture of the simulation environment is shown in Fig. 5. Webots simulates hydrodynamic forces, noise in the actuators as well as inertial and GNSS measurements. The kinematics of the AUVs in simulation were roughly 


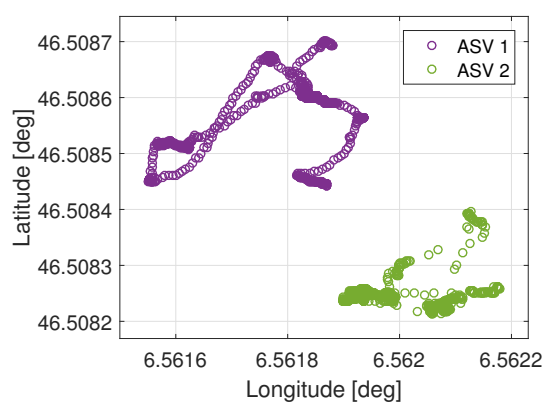

(a) ASV locations

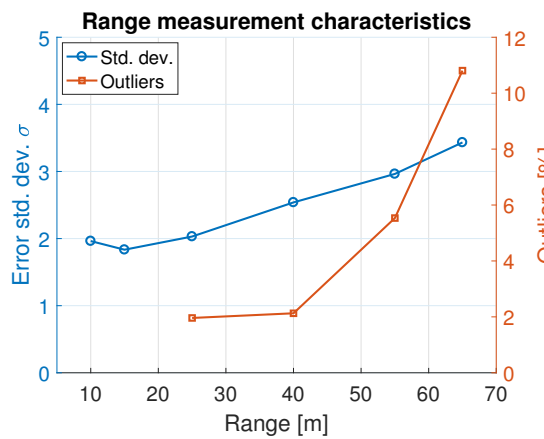

(b) Ranging error and outliers

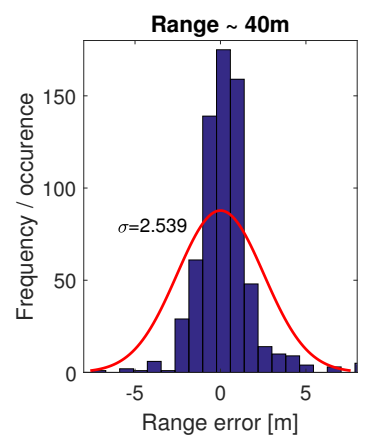

(c) Range error dist. at $=40 \mathrm{~m}$.

Fig. 6: (a) A part of the ASV locations at which range measurements were recorded. (b) Characteristics of error in range measurements, showing standard deviation in the error and occurrence of outliers for various values of range. Outlier occurrence is shown as a percentage of the total number of range measurements performed. (c) Distribution of error in range and bearing measurements at $40 \mathrm{~m}$ range, with a Gaussian fit, after outlier rejection.

\begin{tabular}{|l|l|}
\hline Config. & Description \\
\hline 2SB-A & Two static beacons at position set A \\
\hline 2SB-B & Two static beacons at position set B \\
\hline 1M.O & One mobile beacon with optimal position based motion \\
\hline 1S1M.O & One static beacon, one mobile beacon (optimal .. motion) \\
\hline 2M.F & Two mobile beacons with formation based motion \\
\hline 2M.O & Two mobile beacons with optimal position based motion \\
\hline
\end{tabular}

TABLE I: Description of various beacon configurations. Positions of static beacons are shown in Fig. 7.

calibrated to that of real AUVs. Note that in order to evaluate the proposed algorithm, we do not rely on an accurate simulation of dynamics of the robots, but only that of kinematics and acoustic interaction between ASVs and AUVs. Acoustic transceivers are not inherently simulated in Webots. To overcome this limitation, we characterized their behavior empirically, and simulated them with real-world propagation delay and ranging accuracy within Webots.

\section{B. Acoustic range measurement characteristics}

To characterize the behavior of the acoustic transceivers, we performed a series of measurements near the shore in Lake Geneva. We mounted the acoustic transceivers on two ASVs, and recorded range measurements as well as GNSS positions by placing both ASVs at a variety of locations shown in Fig. 6a. Note that due to forces from wind and waves, it is not possible to hold the ASV static. We then measured the accuracy with respect to GNSS and frequency of occurrence of outliers. The GNSS itself has a positioning error of about $1 \mathrm{~m}$. We found that with increasing distance, the error in range measurements as well as the occurrence of outliers increases significantly. The accuracy characteristics, are shown in Figs. $6 \mathrm{~b}$ and $6 \mathrm{c}$. Note that these measurements were performed in a shallow region of the lake, where the depth of the water column ranges between $5-15 \mathrm{~m}$. We used these characteristics to generate realistic range measurements with additive Gaussian noise in the Webots simulator.

\section{Simulation scenarios}

We simulated various beacon configurations with different AUV operation scenarios. We used a combination of static

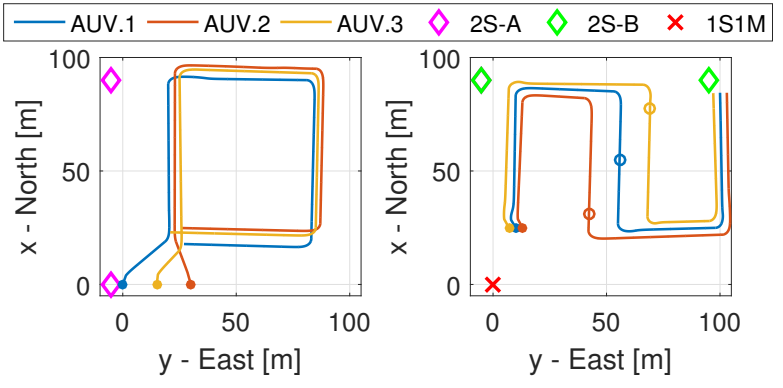

Fig. 7: Preplanned sets of trajectories T1 (left) and T2 (right). The starting point is shown with a dot. The circles in $\mathrm{T} 2$ indicate the positions of the three AUVs at a particular instant of time. The position of static beacons in various configurations is also shown. Experiments with one AUV use only AUV.1 trajectory. Note that we also perform experiments with the combination ' $2 \mathrm{~S}-\mathrm{A}$ ' with $\mathrm{T} 2$ and vice-versa.

and mobile beacons. Experiments were performed with a single as well as a group of three AUVs, with two different sets of trajectories. The AUV trajectories were pre-planned (but the beacons had no prior knowledge of it). The trajectories and static beacon locations are shown in Fig. 7. A summary of the various beacon configurations is provided in Table I. In optimal beacon positioning configurations ('xx.O'), the optimal positions were calculated using the strategy in Section III-D. In the formation configuration ('2M.F'), the two beacons maintained a fixed relative position with respect to the centroid of the AUV group.

We set the maximum speed of the AUVs to $1 \mathrm{~m} / \mathrm{s}$ and that of the mobile beacons to $2.5 \mathrm{~m} / \mathrm{s}$. The maximum separation between the AUVs, $d_{\max }$ in these trajectories was $25 \mathrm{~m}$. By exploiting motion, we aim to place the beacons such that the maximum range measurement, $r_{\max }<50$ for low noise (although the rated maximum range of the DiveNET transceivers is about $1 \mathrm{~km}$ ). We compared the accuracy of the estimated trajectory for each combination of AUV operation scenario and beacon configuration.

\section{RESUlts}

\section{A. Evaluation metric}

We use the Root Mean Squared Error (RMSE) as a measure of accuracy. We compute it as the Euclidean distance between the true and estimated positions averaged over all 


\begin{tabular}{|l|cccccc|}
\hline & \multicolumn{7}{|c|}{ RMSE [m] } \\
\hline Scenarios & 2S-A & 2S-B & 1 M.O & 1S1M.O & 2M.F & 2M.O \\
\hline T1, 1AUV & 3.45 & 3.49 & 2.18 & 2.50 & 2.04 & $\mathbf{1 . 8 7}$ \\
\hline T2, 1AUV & 3.81 & 4.34 & 2.32 & 2.65 & 2.04 & $\mathbf{1 . 9 4}$ \\
\hline T1, 3AUV & 4.54 & 4.78 & 3.56 & 3.61 & 3.10 & $\mathbf{2 . 9 8}$ \\
\hline T2, 3AUV & 4.57 & 4.64 & 3.09 & 3.50 & 2.98 & $\mathbf{2 . 7 7}$ \\
\hline
\end{tabular}

TABLE II: RMSE of the estimated trajectory in various scenarios. $\mathrm{S}=$ static beacon, $\mathrm{M}=$ mobile beacon, $\mathrm{F}=$ motion in fixed formation, $\mathrm{O}=$ motion towards optimal beacon position.

time steps since the beginning of the experiment.

$$
\operatorname{RMSE}(\mathrm{T})=\frac{1}{T} \sum_{t=1}^{T}\left\|x^{t}-\hat{x}^{t}\right\| .
$$

RMSE is higher when the estimated trajectory is farther off from the true trajectory. The RMSE of the estimated trajectories in various scenarios averaged over 5 runs are shown in Table II.

\section{B. Two static beacons}

An example of a trajectory estimated with two static beacons is shown in Fig. 8a. Static beacons result in decreasing measurement accuracy as the AUVs move further from the beacons. Further, a bad combination of relative positions between the beacons and the AUVs (such as collinearity) result in inaccurate position estimates. This can be seen in the upper segment of the trajectory in Fig. 8a. As a result, the two static beacons result in the highest estimated error in most experimental scenarios (see 2S-A and 2S-B in Table II).

\section{One mobile beacon}

We performed experiments with a single mobile beacon using the proposed approach (1M.O in Table II). In order to show that successive optimal beacon locations can be diverse and to stress importance of having multiple beacons, we set the beacon velocity to a hypothetically large value of $4 \mathrm{~m} / \mathrm{s}$. We found that the beacon was not able to reach the optimal locations in time, owing to large distances between successive optimal locations, despite the higher velocity. The estimated trajectory of the AUV, as well as that of the mobile beacon in a one AUV experiment is shown in Fig. 8b. A subset of the optimal locations obtained by solving Eq. (16) are also indicated, showing large variation in successive locations. This generally provides an improvement in accuracy as compared to two static beacons, but a lower accuracy compared to two mobile beacons.

We also used one mobile beacon in combination with one static beacon (1S1M.O). While this reduces the amount of movement required by the mobile beacon to some extent, the mobile beacon still misses some of the calculated optimal locations. Estimated AUV trajectories in a three AUV experiment with this beacon configuration are shown in Fig. 9a.

\section{Two mobile beacons}

We found that two mobile beacons generally outperformed other beacon configurations. The proposed optimal approach
(2M.O) resulted in the lowest RMS error in all the scenarios. However, fixed formation motion (2M.F) had only slightly higher an aggregate error in many scenarios, and a lower error in some individual runs of the simulation. This is because the constant velocity assumption yields poor prediction of future AUV positions (and uncertainties) in case an AUV changes direction shortly after the prediction is made. This results in a sub-optimal beacon placement. Regardless of the relative positions between the beacons and AUVs, closer distance provides more accurate range measurements, due to the characteristics of the error in range measurements. Fig. 8c shows a plot of the trajectory estimated with range measurements from two mobile beacons using the proposed approach in a one AUV experiment. Estimated trajectories in a three AUV experiment with two mobile beacons in formation (2M.F) are shown in Fig. 9b, while those with the two beacons using the proposed method (2M.O) are shown in Fig. 9c.

\section{Conclusion AND OUTLOOK}

This paper presents an approach for repeated, optimal placement of mobile acoustic beacons providing range measurements to a group of AUVs. The resulting mobile beacon trajectory follows the motion of the AUVs. It also provides range measurements from a direction that is close to the direction of maximum uncertainty in position of the AUVs. Our approach addresses the problem of limitation in operational area imposed by fixed, static beacons. It also avoids the problem of collinearity. Our approach is especially useful in shallow water and in coastal areas, where error in range measurements increases significantly with distance due to reflections and occlusions caused by nearby obstacles.

Ranging systems based on two-way transmissions, such as the DiveNET system used in this work, require dedicated time-slots for each AUV. This limits the scalability of the system, because the time required to serve all the AUVs increases linearly with the number of AUVs. In order to achieve the best results with a large number of AUVs, a tradeoff needs to be made between how often to reevaluate optimal positions and which AUVs to serve first. Ranging systems based on one-way-travel-time would be more efficient, since the localization targets are passive receivers, much like GNSS receivers (although they would still need to broadcast their position estimates). However, they require accurate time synchronization, which introduces complexity.

A number of improvements to the system are possible. The beacons rely on position estimates reported by the AUVs to track them. Technically, it is possible for two or more beacons to track the AUVs accurately based on their acoustic transmissions using triangulation. However, this is not inherently supported by the hardware used in this work. Further, the beacons assume a constant velocity (and heading) to predict future positions and uncertainty of the AUVs. A better prediction can be made by sharing additional information, such as a future waypoint. 
